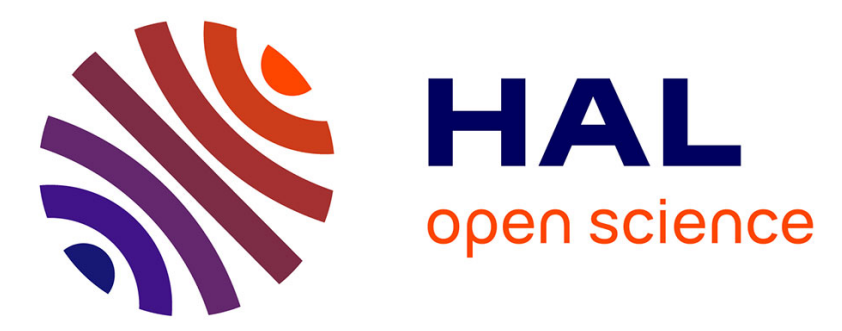

\title{
Vers des Agents Conversationnels Animés Socio-Affectifs
}

Magalie Ochs, Yu Ding, Nesrine Fourati, Mathieu Chollet, Brian Ravenet, Florian Pecune, Nadine Glas, Ken Prepin, Chloé Clavel, Catherine Pelachaud

\section{To cite this version:}

Magalie Ochs, Yu Ding, Nesrine Fourati, Mathieu Chollet, Brian Ravenet, et al.. Vers des Agents Conversationnels Animés Socio-Affectifs. 25ème conférence francophone sur l'Interaction Homme-Machine, IHM'13, AFIHM, Nov 2013, Bordeaux, France. 10.1145/2534903.2534913 . hal$00877271 \mathrm{v} 2$

\section{HAL Id: hal-00877271 \\ https://hal.inria.fr/hal-00877271v2}

Submitted on 6 Nov 2013

HAL is a multi-disciplinary open access archive for the deposit and dissemination of scientific research documents, whether they are published or not. The documents may come from teaching and research institutions in France or abroad, or from public or private research centers.
L'archive ouverte pluridisciplinaire HAL, est destinée au dépôt et à la diffusion de documents scientifiques de niveau recherche, publiés ou non, émanant des établissements d'enseignement et de recherche français ou étrangers, des laboratoires publics ou privés. 


\title{
Vers des Agents Conversationnels Animés Socio-Affectifs
}

\author{
Magalie Ochs, Yu Ding, Nesrine Fourati, Mathieu Chollet, Brian Ravenet, \\ Florian Pecune, Nadine Glas, Ken Prepin, Chloé Clavel et Catherine Pelachaud \\ CNRS-LTCI, Télécom ParisTech, Paris, France \\ greta@telecom-paristech.fr
}

\begin{abstract}
RÉSUMÉ
Dans cet article, nous proposons une architecture d'un Agent Conversationnel Animé (ACA) socio-affectif. Les différents modèles computationnels sous-jacents à cette architecture, permettant de donner la capacité à un ACA d'exprimer des émotions et des attitudes sociales durant son interaction avec l'utilisateur, sont présentés.
\end{abstract}

\section{Mots Clés}

Agent Conversationnel Animé ; émotions ; relations sociales, attitudes sociales.

\section{ACM Classification Keywords}

H.5.1. Information interfaces and presentation (e.g., HCI): Multimedia Information Systems.

\section{INTRODUCTION}

Aujourd'hui, l'essor des nouvelles technologies place les systèmes interactifs dans des rôles de plus en plus variés, typiquement incarnés par des humains, tels que celui de tuteur, de compagnon de jeu, ou encore de conseiller. Lorsque ces systèmes interactifs sont dotés de caractéristiques de la communication humaine, les utilisateurs tendent à interagir naturellement et socialement avec ces derniers comme s'ils étaient humains [31]. Ce rapprochement de la communication humaine dans les interfaces virtuelles peut être atteint en utilisant des artefacts humanoïdes capables de simuler la richesse de la conversation humaine. Les récents progrès dans les technologies informatiques ont rendu possible la création de ces artefacts, appelés Agents Conversationnels Animés (ACAs). Un agent conversationnel animé est un personnage virtuel créé par l'ordinateur qui peut converser avec les utilisateurs d'une façon naturelle à travers un langage verbal et nonverbal [10]. Dans cet article, nous considérons les personnages virtuels humanoïdes mais les ACAs peuvent s'incarner sous d'autres formes plus ou moins réalistes.

Pour concevoir des ACAs capables d'incarner différents rôles efficacement lors d'une interaction avec

(C) ACM, 2013. This is the author's version of the work. It is posted here by permission of ACM for your personal use. Not for redistribution. The definitive version was published in Actes de la $25^{\text {ième }}$ conférence francophone sur l'Interaction Homme-Machine, 2013.

http://dx.doi.org/10.1145/2534903.2534913 l'utilisateur, à l'image de l'humain, ces derniers doivent être dotés d'une certaine forme d'intelligence socioémotionnelle [29, 45]. Cette forme d'intelligence doit leur permettre de gérer la dimension intrinsèquement sociale et émotionnelle de l'interaction humain-machine en adoptant un comportement émotionnel et une attitude sociale adaptés au contexte de l'interaction.

Un certain nombre de recherches montrent en effet que les ACAs dotés de capacités socio-émotionnelles permettent d'améliorer significativement l'interaction [4]. Pour créer des agents avec lesquels l'utilisateur s'engage dans une interaction à plus ou moins longterme, ces derniers doivent intégrer la subtilité expressive de l'humain. Par exemple, pour que leurs comportements émotionnels paraissent crédibles, les ACAs devraient être capables d'exprimer des émotions aussi bien lorsqu'ils écoutent que en action, i.e. lorsqu'ils parlent ou lorsqu'ils réalisent une action particulière [14]. Pour ce faire, dans la section «Expression d'émotions d'un ACA », des modèles d'expressivités émotionnelles faciales et corporelles que nous avons construit à partir de corpus d'individus exprimant des émotions, sont présentés. De plus, les ACAs devraient pouvoir être capable d'exprimer leur attitude vis-à-vis de l'utilisateur que ce soit de l'appréciation ou de l'hostilité, non pas seulement à travers des mots mais aussi à travers leur comportement non-verbal. Pour répondre à cette problématique, dans la section «Expressions intra-personnelles des attitudes sociales d'un ACA », nous présentons un modèle computationnel, que nous avons construit à partir d'une approche centrée sur la perception de l'utilisateur, pour déterminer le comportement non-verbal d'un ACA suivant son attitude sociale. En dehors de l'aspect expressif, les ACAs devraient être capables de raisonner sur le contexte de l'interaction pour déterminer quel comportement socio-émotionnel adopter et montrer dans quelle situation. Pour calculer les émotions que l'ACA devrait exprimer, nous utilisons un modèle existant présenté dans la section «Modèle computationnel d'émotions ». Concernant les attitudes sociales, un premier modèle conceptuel pour la représentation et le raisonnement est proposé dans la section «Modèle computationnel des attitudes sociales ». Impliqué dans une interaction, ce comportement de l'agent devrait considérer le comportement socio-émotionnel exprimé par l'utilisateur et la relation qui se construit entre l'ACA 
et l'utilisateur. Pour ce faire, le raisonnement de l'ACA intègre un réseau de neurones que nous avons développé, considérant les signaux non-verbaux exprimés par l'interlocuteur (Section «Expressions inter-personnelles des attitudes sociales »).

Dans cet article, nous proposons une architecture intégrant ces différentes capacités cognitives, expressives et interactives d'un ACA socio-affectif ainsi que les différents modèles computationnels sous-jacents permettant de doter un ACA de telles capacités. Plusieurs ACAs intégrant une dimension sociale ou émotionnelle ont d'ores et déjà été proposés (e.g. $[6,18,20,31])$. L'enjeu des travaux de recherche présentés dans cet article est l'articulation de différents modèles au sein d'une même architecture pour doter l'ACA à la fois d'émotions (1ère section) et d'attitudes sociales (2ème section). Les pistes de recherche envisagées au sein de cette architecture sont ensuite présentées en dernière section.

\section{ARCHITECTURE D'UN ACA SOCIO-AFFECTIF}

L'architecture d'un ACA, illustrée Figure 1, correspond à une extension de l'architecture SAIBA. L'architecture SAIBA [30] est une architecture de référence dans le domaine des ACAs. Cette architecture unifiée est indépendante du domaine d'application, du système d'animation et de l'environnement graphique. Elle permet la génération de comportements multimodaux d'ACA. Dans SAIBA, le planificateur d'intentions génère les intentions communicatives de l'ACA (par exemple, l'intention d'exprimer de la joie ou son accord). Le planificateur de comportements transforme ces intentions communicatives en un ensemble de signaux (e.g. voix, gestes, expressions faciales). Par exemple, ce module fournit en sortie les signaux que devraient exprimer l'ACA suivant ses émotions, son attitude et son intention (Section «Expressions corporelles affectives» et «Expressions intrapersonnelles des attitudes sociales »). La liste des signaux pouvant être utilisés pour exprimer chaque intention communicative est définie dans le lexique de comportements. Les caractéristiques morphologiques et dynamiques d'un signal pour animer le visage (par exemple un sourire) sont décrites dans le module d'expressions faciales. Les expressions multimodales (expressions et synchronisation des signaux sur différentes modalités) sont gérées par le planificateur de comportements. Enfin, le module réalisation de comportements fournit en sortie les paramètres d'animation pour chacun des signaux. Par exemple, ce module produit en sortie les paramètres d'animation des sourcils et des mouvements de tête suivant l'émotion de l'ACA (Section « Expressions faciales affectives »).

L'architecture SAIBA est étendue avec différents modules pour ajouter certains raisonnements cognitifs de l'agent et la prise en compte de la dimension interactive. Le module Etat mental contient une représentation des paramètres socio-affectifs de l'agent tels que ses émotions, ses relations sociales, ses buts et ses croyances. Il contient de plus des modèles de raisonnement permettant de calculer la dynamique de ses paramètres au cours de l'interaction. Les émotions et les relations sociales sont calculées par des modèles intégrées dans l'Etat mental (Section «Modèle computationnel des émotions » et Modèle computationnel des attitudes sociales »).

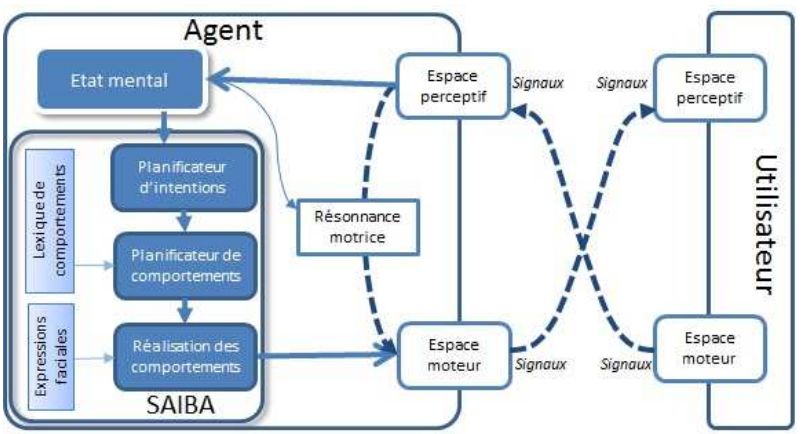

Figure 1 : Architecture d'un ACA socio-affectif

Les modules Espace perceptif, Résonnance motrice et Espace moteur ont été ajoutés pour permettre de simuler l'influence directe (i.e. par résonnance motrice, influence illustrée par les flèches en pointillées Figure 1) du comportement socio-affectif de l'utilisateur sur le comportement de l'agent. Ces modules permettent en particulier à l'ACA d'aligner son comportement sur celui de son interlocuteur, par exemple par mimétisme, pour traduire une attitude sociale spécifique. Ces modules sont présentés plus en détails dans la section « Expressions inter-personnelles des attitudes sociales ».

A ce jour, cette architecture a été partiellement implémentée et évaluée [33]. Certains modules sont en cours d'intégration. Dans la suite de l'article, nous décrivons plus précisément les différents modèles sousjacents à cette architecture.

\section{L'EXPRESSION D'EMOTIONS D'UN ACA}

L'interaction humain-humain est, d'une manière générale, réalisée à travers différentes modalités telles que la parole, les gestes, les sourcils, la tête, le regard, etc. Les variations dans ces modalités permettent d'augmenter la compréhension de la parole. De plus, ces comportements non-verbaux traduisent des sentiments subtils tels que les émotions. Pour donner la capacité à un ACA d'exprimer des émotions, il est donc essentiel que ces derniers soient capables de générer des comportements non-verbaux naturels et crédibles lors d'une conversation avec un humain.

\section{Expressions faciales affectives}

Dans les relations interpersonnelles, les émotions sont principalement communiquées par l'expression faciale [22]. Une expression faciale est un changement dans le visage, perceptible visuellement suite à l'activation (volontaire ou involontaire) des muscles composant le visage. Aujourd'hui, de nombreux ACA sont capables 
d'exprimer des émotions à travers leurs expressions faciales (par exemple, [18]). Cependant, très peu d'entre eux sont capables d'exprimer une émotion lorsqu'ils parlent. Pour ce faire, les ACAs doivent pouvoir synchroniser la parole et l'expression faciale affective.

Pour calculer automatiquement le comportement nonverbal d'un ACA synchronisé avec la parole, différentes méthodes ont été proposées. Une méthode consiste à utiliser la capture de mouvement. Cependant, cette méthode ne peut être utilisée que pour un ensemble fini de phrases (celles dont les mouvements sont capturés). Une autre méthode repose sur des règles prédéfinies. Par exemple, dans [11], les comportements sont générés selon les relations identifiées entre la prosodie de la parole et les comportements non-verbaux. Cependant, ces règles ne considèrent pas l'influence des émotions sur ces relations. Depuis une dizaine années, des modèles statistiques d'apprentissage ont été proposés pour synthétiser automatiquement divers comportements à partir de la parole synchronisée. Par exemple, dans [8], les auteurs ont proposé d'apprendre séparément quatre modèles utilisés pour synthétiser les expressions de tristesse, de satisfaction ou de colère de manière synchronisée avec la parole. Le nombre d'émotions reste cependant très limité. De plus, les modèles proposés ne permettent généralement pas de considérer l'expression de plusieurs émotions simultanément [22].

Afin de donner la capacité à un ACA d'exprimer plusieurs émotions, nous avons développé un modèle statistique par apprentissage pour synthétiser automatiquement les mouvements des sourcils et de la tête à partir du signal de la parole. Ce modèle repose sur un modèle de Markov caché entièrement paramétré (fully parameterized HMM - FPHMM). Le modèle est décrit plus en détails dans [21]. Ce modèle a été appris à partir des données d'un corpus audiovisuel contenant des vidéos d'acteurs équipés de microphones et de capteurs de mouvements du visage [23]. Les acteurs avaient comme instructions de prononcer différentes phrases avec diverses émotions. Trois angles de rotation de tête et huit points d'animation faciale (Facial animation Points - FAPs, [37]) ont été extraits comme caractéristiques du mouvement avec une fréquence de $25 \mathrm{~Hz}$. Les caractéristiques de la parole, extraites avec les mêmes fréquences d'échantillonnage de $25 \mathrm{~Hz}$ sont la fréquence fondamentale et l'énergie du signal de parole. Pour la synthèse des expressions faciales affectives d'un ACA, la séquence de signaux de parole est utilisée en entrée du modèle FPHMM qui produit en sortie les mouvements de sourcils et de tête associés.

Pour évaluer ce modèle, des tests perceptifs ont été réalisés. Nous avons comparé la perception des expressions d'émotions d'un ACA résultantes du modèle FPHMM produit et celles résultantes directement de la capture de mouvement. Douze émotions ont été considérées (colère, tristesse, peur, mépris, nervosité, dégout, frustration, stress, excitation, confiance, surprise et joie) ${ }^{1}$ et exprimées durant la prononciation de différentes phrases. Quarante utilisateurs ont indiqué leur perception des émotions ainsi exprimées. Les analyses statistiques montrent que le modèle proposé permet d'améliorer significativement la perception des émotions de peur, mépris, nervosité, stress, excitation surprise et joie en comparaison avec les expressions d'ACA générées à partir des données de capture de mouvements directement. Les résultats de l'évaluation sont détaillés dans [21].

Ce modèle s'intègre dans le module de réalisation de comportements de l'architecture d'un ACA (Figure 1). Il permet de calculer les paramètres d'animation pour les sourcils et les mouvements de tête à partir du signal de parole de l'ACA issu de la sortie de la synthèse vocale.

\section{Expressions corporelles affectives}

Les émotions sont exprimées non seulement à travers l'expression faciale mais aussi à travers l'ensemble du corps. Dans un ACA, l'expression des émotions à travers ces différentes modalités permet d'améliorer la perception des émotions par l'utilisateur ainsi que la crédibilité de l'agent [14].

La synthèse des comportements émotionnels chez un ACA implique deux principales problématiques: la définition d'un système de codage des mouvements corporels (i.e. comment représenter les mouvements corporels) et la correspondance entre ce système de codage et les états affectifs (i.e. comment l'émotion est exprimée à travers ces mouvements). Certains travaux utilisent un système de codage corporel basé sur le type de mouvement [15]. Les modèles de comportement émotionnel sont donc définis à travers un ensemble discret de postures et/ou d'actions, comme le croisement des bras ou la flexion de la tête. Dael et al. [17] ont récemment proposé un nouveau système de codage des mouvements corporels basé sur le type de mouvement. Ce système décrit le mouvement du corps en termes d'unités d'action et de posture en tenant compte des différents niveaux des dimensions anatomique et directionnel. Les modèles de comportements émotionnels corporels basés sur le type de mouvement sont donc basés sur les travaux précédents portant sur l'expression d'émotion à travers les mouvements du corps [48]. Une autre méthode consiste à utiliser une base de données de capture de mouvements émotionnels naturels ou actés dans le but d'en extraire des informations utiles pour la reproduction $\mathrm{du}$ comportement expressif et émotionnel chez un ACA. Dans cette approche, les mouvements corporels sont décrits par des paramètres définissant la qualité du mouvement, i.e. la manière avec laquelle le mouvement a été effectué. Dans les travaux existants, les paramètres

\footnotetext{
${ }^{1}$ Ces émotions correspondent à celles considérées dans le corpus [23].
} 
qui contrôlent l'expressivité du mouvement se limitent généralement à un ensemble de paramètres d'expressivité calculés à partir de données de capture de mouvement pour un type de geste particulier [43]. Cependant, ces paramètres sont souvent associés à un nombre limité de types de geste et ne peuvent pas être étendus à l'ensemble des gestes possibles d'un ACA. D'autres travaux proposent de contrôler l'expressivité des mouvements de l'ACA (par exemple leur amplitude) à travers des fonctions mathématiques reposant sur quelques lois invariantes du mouvement humain [28]. Ce type d'approche permet d'appliquer les mêmes paramètres pour plusieurs types de gestes. Cependant, l'ensemble des paramètres reste limité agissant principalement sur les gestes des mains.

Nous proposons un modèle de comportement émotionnel reposant sur l'analyse d'un corpus de capture de mouvements. Inspiré du système de codage défini dans [17], la définition des paramètres que nous avons adoptée pour contrôler le comportement émotionnel corporel d'un ACA repose sur plusieurs niveaux de description, à la fois pour décrire un mouvement corporel et pour décrire les caractéristiques de ce mouvement. Trois principales dimensions sont considérées: une dimension anatomique définissant les articulations en questions (e.g. la tête, les bras), une dimension directionnelle définissant la direction du mouvement (e.g. latérale, verticale) et une dimension statique/dynamique décrivant l'aspect postural ou dynamique du geste (e.g. la posture statique, le changement de posture au cours du temps). Une première étude que nous avons réalisée [24], utilisant une partie de l'ensemble des paramètres pour analyser des marches expressives, a montré qu'on peut discriminer différents styles de marche à travers quelques caractéristiques décrivant la posture et le changement de posture au cours du temps. La marche de tristesse est par exemple caractérisée principalement par le fléchissement des coudes, l'absence d'une variation significative de la distance entre les mains, le fléchissement de la tête et l'effondrement du torse.

Nous poursuivons actuellement nos travaux à partir d'une base de données de captures de mouvements de comportements émotionnels actés que nous avons collectés. Onze acteurs ont exprimé 3 fois 8 états affectifs différents (tristesse, colère, joie, honte, fierté, anxiété, peur panique, et neutralité) en réalisant 7 actions différentes. L'objectif est d'étendre l'étude décrite cidessus sur la marche à d'autres mouvements tels que frapper à la porte ou déplacer des objets sur une table. Les comportements émotionnels corporels modélisés à partir de la base de données seront intégrés dans le module Planificateur de comportements de l'architecture de l'ACA illustrée Figure 1. Ainsi, les paramètres des mouvements de l'ACA pour réaliser une action pourront être calculés suivant l'émotion de ce dernier.

\section{Modèle computationnel d'émotions}

Outre la dimension expressive, un ACA doit être capable de déterminer quelles émotions exprimer durant une interaction avec l'utilisateur. Les recherches ont en effet montré qu'une même émotion exprimée par un ACA dans une situation inappropriée pouvait avoir un effet néfaste sur l'interaction [4]. Fondés pour la plupart sur les théories de l'évaluation cognitive des émotions [46], un certain nombre de modèles computationnels a d'ores et déjà été proposé pour calculer et mettre à jour automatiquement les émotions de l'ACA suivant le déroulement de l'interaction. Dans l'architecture de l'ACA socio-affectif présentée Figure 1, le modèle computationnel FAtiMA [20] a été intégré dans le module Etat mental. Le modèle FAtiMA calcule les émotions de l'ACA suivant ses croyances sur l'état de ses buts et ses besoins. Ce modèle présente de plus l'avantage d'offrir une architecture modulaire permettant de simuler des phénomènes tels que la culture ou l'empathie dans le processus de déclenchement des émotions de l'agent.

En dehors de la dimension émotionnelle, un ACA étant engagé dans une interaction sociale avec l'utilisateur, doit être capable d'exprimer différentes attitudes vis-àvis de ce dernier. Dans la section suivante, nous présentons des modèles permettant de doter l'ACA de telles capacités.

\section{LES ATTITUDES SOCIALES D'UN ACA}

Durant une interaction, suivant la relation entre deux personnes et le contexte (e.g. leurs rôles et leurs intentions), celles-ci ne vont pas exprimer la même attitude sociale. Scherer [46] définit l'attitude sociale comme «an affective style that spontaneously develops or is strategically employed in the interaction with a person or a group of persons ». Les attitudes sociales sont souvent représentées sur deux dimensions, la dominance et l'appréciation. Cette représentation, proposée par Argyle [2], est généralement illustrée sous forme d'un disque appelé le circomplexe interpersonnel (Interpersonal Circumplex) où une attitude sociale correspond à un point dans cet espace à deux dimensions.

Lors d'une interaction, de nombreux éléments du comportement non-verbal reflètent l'attitude sociale d'un individu [7]. Lorsqu'un message verbal est transmis, les caractéristiques des signaux non-verbaux qui l'accompagnent peuvent traduire l'attitude sociale du locuteur. Par exemple, une attitude dominante se traduit par des gestes amples [9]. De plus, la dynamique intrapersonnelle, i.e. la temporalité des signaux exprimés par un même individu sur un tour de parole ou sur l'ensemble de l'interaction, caractérise une certaine attitude. Par exemple, de fréquents sourires peuvent correspondre à une attitude d'appréciation. Enfin, dans une interaction, l'attitude est exprimée à travers la dynamique inter-personnelle, i.e. la temporalité des signaux d'un individu par rapport à celui de son 
interlocuteur. Par exemple, un sourire exprimé en réponse à un sourire de l'interlocuteur peut exprimer de l'affiliation [32].

Dans cette section, nous présentons tout d'abord des modèles permettant de déterminer comment un ACA peut exprimer une attitude sociale à travers le comportement non-verbal qui accompagne un message verbal et à travers la dynamique intra- et interpersonnelle de son comportement non-verbal. Nous présentons ensuite un modèle permettant de déterminer quelle attitude sociale l'ACA devrait exprimer durant une interaction.

\section{Expressions intra-personnelles des attitudes sociales d'un ACA}

Différents travaux de recherche ont été menés afin de doter les ACAs de la capacité d'exprimer des attitudes sociales à travers leurs comportements non-verbaux. Par exemple, l'ACA Laura [6] montre son appréciation visà-vis de l'utilisateur par une modification de certains paramètres comme l'augmentation du nombre de hochements de tête et de gestes. Dans le projet Demeanour [26], les agents sont capables de changer certains paramètres de leur comportement non-verbal (la posture, les regards, et l'activation des gestes) suivant leurs attitudes représentées à l'aide de circomplexe interpersonnel.

Afin de créer un ACA capable de montrer différentes attitudes sociales à travers différentes modalités, nous avons utilisé une méthode fondée sur la perception de l'utilisateur. Nous avons collecté un corpus d'ACAs (féminin et masculin) exprimant différentes attitudes sociales (dominant, soumis, amical et hostile) en demandant à l'utilisateur de directement créer le comportement non-verbal associé. Nous avons, pour ce faire, développé et mis en ligne une application qui permet à un utilisateur d'aisément configurer les paramètres d'animation du comportement non-verbal d'un ACA pour une attitude et une intention communicative données (Figure 2).

Nous avons ainsi collecté 925 descriptions de comportements non-verbaux (170 participants). Chaque comportement du corpus est décrit par une attitude (dominant, soumis, amical et hostile), une intention communicative (informer, demander, accepter, refuser, approuver, désapprouver, que nous utilisons pour illustrer la situation), et un ensemble de valeurs pour les paramètres suivant du comportement non-verbal: la présence d'évitement de regard, la modalité utilisée (mouvement de tête, des bras, les deux ou aucun mouvement), l'expression faciale (positive, négative ou neutre), l'amplitude et la force des gestes (faible, moyenne ou forte) et l'orientation de la tête (droite, vers le bas, vers le haut ou sur le côté). Ces paramètres ont été choisis au regard de la littérature en Sciences Humaines et Sociales montrant une influence des attitudes sur ces modalités. Chaque participant avait comme tâche de configurer le comportement de l'ACA dans 6 situations différentes (une situation correspondant à une intention communicative et une attitude sociale).

A partir des données collectées, pour pouvoir calculer automatiquement les paramètres du comportement d'un ACA suivant l'attitude sociale qu'il souhaite exprimer, nous avons développé un modèle fondé sur un réseau Bayésien. Dans ce modèle, la structure des arcs entre les variables d'entrée (l'attitude et l'intention) et les variables de sorties (les paramètres du comportement non-verbal), est construite suivant les corrélations entre celles-ci, calculées statistiquement à partir des données du corpus (les résultats statistiques sont décrits plus précisément dans [41]). Les données du corpus sont de plus utilisées pour calculer les paramètres du modèle, i.e. les probabilités conditionnelles des valeurs des variables.

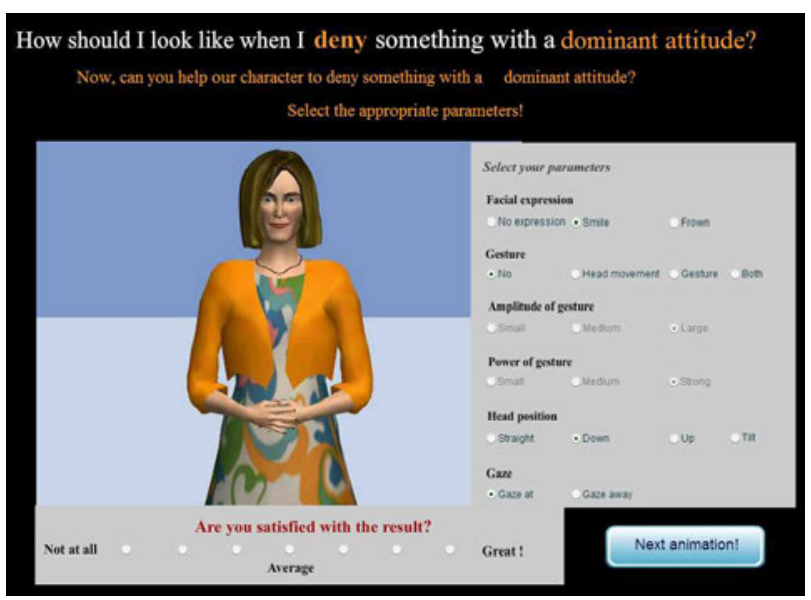

Figure 2: Copie d'écran de l'interface de collecte de comportements non-verbaux d'ACA

Ce modèle permet à un ACA qui doit exprimer une intention communicative avec une certaine attitude sociale, de choisir le comportement non-verbal correspondant [41]. Il s'intègre dans l'architecture de l'ACA (Figure 1) dans le module Planificateur de comportements pour la sélection du choix des signaux.

Actuellement, nous travaillons sur l'extension de ce modèle bayésien pour l'étendre à l'influence d'autres intentions communicatives sur les paramètres du comportement non-verbal. L'objectif est que ce modèle puisse déterminer automatiquement par quel comportement non-verbal l'ACA peut montrer une attitude à partir d'une description du contexte socioaffectif du dialogue et des intentions communicatives.

L'attitude sociale se traduit non seulement à travers l'expression d'une intention communicative à un instant $t$ mais aussi à travers la dynamique des signaux dans un tour de dialogue ou tout au long de l'interaction, i.e. la dynamique intra-personnelle du comportement nonverbal. Par exemple, de fréquents sourires suivis par une absence totale de sourire peuvent traduire une attitude d'hostilité. 
Afin de modéliser la dynamique intra-personnelle des comportements non-verbaux qu'un ACA devrait adopter pour exprimer une attitude sociale particulière, nous proposons d'analyser des corpus d'interactions interpersonnelles. Cette méthode d'analyse de corpus a d'ores et déjà été explorée mais principalement avec le but de développer des méthodes de détection automatiquement des attitudes sociales des individus. Une revue de ces méthodes existantes de détection automatique d'informations sociales dans des interactions est disponible dans [25]. La plupart des approches existantes se sont concentrées sur l'utilisation de mesures audio-visuelles sur l'ensemble d'une interaction pour classifier la dominance des personnes dans un groupe. Si ces approches se révèlent efficaces dans leur tâche de classification de la dominance d'une personne dans la globalité d'une interaction, elles ne permettent pas de détecter les variations locales d'attitude par rapport à la dynamique des comportements non-verbaux. De plus, il n'y a pas eu, à notre connaissance, de tentative d'intégration de ces résultats dans un modèle de comportement d'ACA.

Afin d'identifier la dynamique des comportements nonverbaux qu'un ACA devrait adopter pour exprimer une attitude durant une interaction, nous avons analysé un corpus multimodal humain-humain composé de 3 vidéos d'entretiens d'embauches, durant en moyenne 20 minutes. Ces vidéos ont été annotées à deux niveaux : (1) au niveau du comportement non-verbal des interactants en s'appuyant sur le schéma de codage MUMIN [1] et (2) au niveau de l'attitude sociale du recruteur. L'attitude sociale a été annotée par 12 personnes en utilisant l'outil GTrace [16] sur les deux dimensions de l'attitude sociale décrites dans la section précédente: la dominance et l'appréciation (une première analyse des annotations est présentée dans [13]). Notre prochaine étape d'analyse vise à étudier les comportements non-verbaux associés aux variations d'attitude sociales annotés dans les vidéos. Pour ce faire, une analyse des séquences de comportements sera réalisée. Cette analyse nous permettra de construire un répertoire des séquences nonverbales permettant à un ACA d'augmenter ou de diminuer son expression de dominance ou d'appréciation. Ces séquences seront intégrées dans le module de Réalisation de comportements (Figure 1) pour modifier le comportement de l'agent suivant les attitudes sociales que devrait exprimer l'ACA.

Dans la section suivante, nous présentons un modèle permettant de modéliser la dynamique inter-personnelle du comportement non-verbal d'un ACA pour exprimer différentes attitudes sociales durant une interaction.

\section{L'expressions inter-personnelles des attitudes sociales de l'ACA}

Durant une interaction, comme le soulignent Chindamo et al. [12] "stances are constructed across turns rather than being the product of a single turn". En effet, lorsque les attitudes de chaque partenaire de l'interaction sont mises en présence, une attitude de la dyade émerge de l'alignement diachronique entre les interactants. Par exemple, l'alignement du comportement non-verbal d'un locuteur et de son interlocuteur (e.g. l'expression de sourires synchronisés) peut traduire une attitude d'intérêt et de plaisir mutuel [32].

Plusieurs agents virtuels capables d'aligner leur comportement non-verbal sur celui de l'utilisateur ont été développés. Par exemple, dans [5], un modèle est proposé pour permettre à un ACA qui écoute l'utilisateur d'automatiquement exprimer des backchannels, i.e. des signaux non-verbaux, comme des hochements de tête ou des sourires, alignés sur ceux de l'utilisateur. Ces travaux montrent que ces signaux de backchannels facilitent en effet l'allocution de l'utilisateur et améliorent sa perception de l'agent. Ces types de modèle proposent une adaptation du comportement non-verbal de l'agent en réaction à celui de l'utilisateur, et non en interaction avec ce dernier. Le comportement de l'agent s'aligne en effet sur celui de l'utilisateur par une expression de signaux de mêmes types que ceux détectés de l'utilisateur. Afin de permettre à un ACA d'aligner dynamiquement son comportement non-verbal en interaction avec celui de l'utilisateur, nous avons développé un modèle permettant de modifier en temps réel les caractéristiques morphologiques et dynamiques des signaux non-verbaux de l'agent suivant les réactions de son interlocuteur. Nous illustrons ce modèle sur le signal du sourire. Lors d'une interaction, si l'ACA sourit à l'utilisateur, et que ce dernier lui sourit en réponse, l'ACA devrait renforcer son sourire pour lui montrer un intérêt et un plaisir partagés [32]. En d'autres termes, l'ACA devrait être capable de moduler dynamiquement son sourire suivant sa perception directe du sourire de son interlocuteur. Pour modéliser le sourire d'un ACA, nous avons fondé nos travaux sur les caractéristiques morphologiques et dynamiques des sourires d'un ACA identifiées dans des travaux précédents [34]. Un sourire est ainsi défini par sa taille (i.e. l'amplitude des muscles zygomatiques), le soulèvement des pommettes, l'ouverture de la bouche et la tension des lèvres. Ainsi, un ACA peut renforcer son sourire en amplifiant la durée et l'amplitude de ces caractéristiques.

Pour permettre à un ACA de modifier dynamiquement et en temps réel son sourire durant une interaction, nous avons développé un modèle computationnel fondé sur un réseau de neurones. Le réseau de neurones permet de définir une architecture neurone par neurone contrôlée dynamiquement. Comme illustré Figure 3, les neurones représentent les caractéristiques du sourire. La couche perceptive (i.e. Espace perceptif) correspond aux caractéristiques perçues alors que la couche générative (i.e. Espace moteur) correspond aux caractéristiques exprimées par l'agent. Les liens entre ces deux couches représentent l'influence des caractéristiques perçues sur celles exprimées (ce modèle est décrit plus en détails dans [39]). 
L'alignement dynamique des sourires de l'ACA, i.e. les liens entre la couche perceptive et générative (le module de Résonnance motrice), vont dépendre de l'attitude sociale que veut exprimer l'agent Cette influence est représentée dans l'architecture de l'ACA (Figure 1) par le lien entre le module Etat mental, et le module Résonnance motrice.

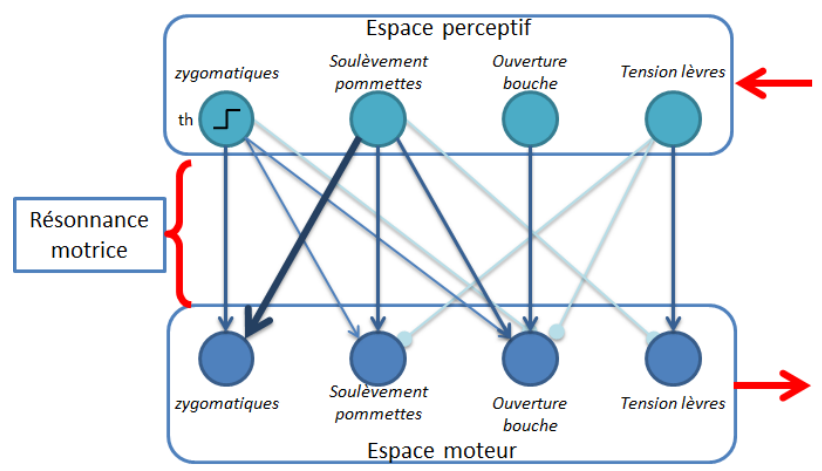

Figure 3: Modèle de résonnance motrice

Ainsi, si l'agent décide de se montrer amical, l'influence sera positive: les sourires de l'ACA seront renforcés par la perception des sourires de l'utilisateur. En d'autres termes, lorsque l'ACA perçoit un sourire de l'utilisateur, suivant les caractéristiques du sourire perçu, l'amplitude et la durée des caractéristiques de son sourire seront augmentées. Dans le cas contraire, les sourires de l'ACA ne seront pas influencés par ceux de l'utilisateur.

Cette modélisation du lien entre les attitudes sociales de 1'ACA et l'alignement de son comportement non-verbal est fondée sur les résultats d'une récente étude perceptive que nous avons réalisée [35]. Dans cette étude, nous avons simulé une interaction entre deux ACAs (dotés du modèle de résonnance motrice décrit ci-dessus) et comparé la perception que les utilisateurs ont de l'ACA lorsque celui renforce ou non son sourire en réponse à celui de son interlocuteur. Les résultats confirment que l'alignement de ce comportement non-verbal a un effet significatif sur la perception de son attitude, le renforcement du sourire améliorant la perception de l'attitude. Nous travaillons actuellement sur l'intégration du système permettant la détection automatique temps réel des caractéristiques des signaux exprimées par l'utilisateur (e.g. les caractéristiques du sourire). La prochaine étape vise à valider, à travers des tests perceptifs, la capacité d'un ACA à exprimer différentes attitudes par l'alignement de son comportement nonverbal (incluant le sourire mais aussi d'autres signaux tels que les hochements de tête), lorsque celui-ci est impliqué dans une interaction directe avec l'utilisateur.

\section{Modèle computationnel des attitudes sociales}

Afin de déterminer l'attitude sociale que devrait exprimer un ACA durant une interaction avec un utilisateur, un modèle computationnel de la dynamique de ces attitudes doit être défini. Comme l'indique Scherer [46], les attitudes sociales sont influencées par différents paramètres qui peuvent être de nature contextuelle, stratégique ou interpersonnelle. Pour qu'un ACA exprime des attitudes sociales appropriées à la situation d'interaction, les mécanismes inhérents aux relations sociales et leur évolution au cours des interactions doivent être modélisés.

Dans le domaine des ACAs, la plupart des modèles computationnels se focalisent sur les relations sociales. Même si ces dernières sont essentielles pour déterminer l'attitude sociale d'un ACA, d'autres éléments du contexte de l'interaction peuvent amener l'ACA à exprimer des attitudes différentes de ces relations « ressenties ». De plus, les modèles existant des relations sociales utilisent principalement les émotions pour calculer la dynamique des relations sociales, par exemple [38]. Comme proposé dans [19], la dynamique peut être aussi modélisée à partir de théories de psychologie cognitive telle que la théorie de la congruité [36] mettant en évidence comment les relations sociales entre les individus s'articulent.

Dans notre modèle, pour calculer l'attitude sociale d'un ACA, nous avons proposé une représentation des différentes relations sociales de l'agent et de l'utilisateur. Comme les attitudes sociales, les relations sociales peuvent être définies selon les deux axes de dominance et d'appréciation, via le circomplexe interpersonnel (décrit précédemment). Dans notre modèle, nous utilisons deux représentations du circomplexe, définissant deux types de relations bien distinctes : pour les relations de l'agent envers l'utilisateur, nous représentons la relation par un vecteur démarrant à l'origine du circomplexe [27]. Les relations de l'utilisateur envers l'agent - qui correspondent aux croyances de l'agent - sont modélisées par un sousensemble du circomplexe prenant ainsi en compte la part d'incertitude relative à ces croyances.

A partir de la définition de Scherer présentée ci-dessus, nous proposons un modèle computationnel dans lequel nous représentons les relations de l'agent envers l'utilisateur par trois vecteurs différents sur ce même circomplexe interpersonnel : (1) la relation sociale ressentie représente le sentiment de l'ACA envers l'utilisateur (e.g. l'agent n'aime pas l'utilisateur). Comme proposé dans [47], ce vecteur de relation sociale est initialisé suivant la personnalité de l'agent; (2) la relation idéale indique la relation que l'agent souhaiterait idéalement exprimer dans une situation particulière (e.g. un enseignant peut vouloir montrer de la dominance envers ses élèves). La taille et la direction de ce vecteur dépendent principalement du rôle de l'agent et de la manière dont l'agent souhaite exprimer ce rôle; (3) l'attitude sociale qui correspond à la relation exprimée par l'agent. Cette attitude est calculée en fonction de la relation ressentie et de la relation idéale. De plus, l'humeur de l'agent influence la valeur d'appréciation (e.g. un agent de mauvaise humeur aura tendance à se montrer plus hostile) [3]. La valeur de dominance est 
aussi influencée par l'état des ressources de l'agent et de l'utilisateur [40].

La dynamique de l'attitude sociale de l'agent, i.e. la relation sociale que l'agent va exprimer durant l'interaction, dépend fortement des croyances de l'ACA sur les relations sociales de l'utilisateur envers l'agent. Le comportement de l'agent va en effet être différent suivant que l'utilisateur montre par exemple de l'appréciation ou de l'hostilité envers lui. Dans notre modèle, les relations de l'utilisateur envers l'agent sont représentées par les 2 relations suivantes : (1) la relation désirée est la relation que l'agent désire voir exprimée chez l'utilisateur. Cette relation est calculée à partir de la relation idéale, en accord avec la théorie de la complémentarité interpersonnelle [44]. D'après cette théorie, la relation désirée est une symétrie de la relation idéale selon l'axe d'appréciation : si l'agent veut montrer de la dominance et de l'appréciation, il s'attend à ce que l'utilisateur exprime de la soumission et de l'appréciation; (2) la relation perçue décrit la relation que l'agent pense que l'utilisateur ressent envers lui (e.g. l'agent pense que l'utilisateur l'apprécie).

Afin de modéliser la dynamique de ces relations et ainsi calculer l'attitude sociale que devrait exprimer l'ACA, notre modèle s'appuie sur la Théorie de la congruité [36]. Nous nous basons donc sur un schéma triangulaire entre un agent $\mathrm{A}$, un utilisateur $\mathrm{U}$ et un concept $\mathrm{C}$. Les arêtes du triangle décrivent la relation de A envers l'utilisateur $\mathrm{U}$ (relation ressentie), la relation de A envers le concept $\mathrm{C}$ (relation idéale) et l'estimation de A sur la relation de $\mathrm{U}$ envers S (relation désirée). Par exemple, l'agent apprécie l'utilisateur, veut montrer de la dominance et s'attend donc à ce que l'utilisateur adopte une attitude soumise. L'interaction est dite congruente quand la relation perçue de $\mathrm{U}$ à propos de $\mathrm{C}$ est en adéquation avec la relation désirée (Figure 3). Si l'on reprend l'exemple précédent, il y a congruence si l'utilisateur montre de la soumission. Dans ce cas, la relation ressentie de l'ACA va tendre vers la relation idéale. En revanche, l'interaction est dite non-congruente si l'agent perçoit de la dominance chez l'utilisateur. L'agent peut alors adopter trois différentes stratégies : (a) il peut changer sa relation ressentie envers l'utilisateur, (b) il peut changer sa propre relation idéale et (c) il peut essayer de changer la relation exprimée par l'utilisateur. Finalement, l'attitude sociale exprimée par l'ACA va dépendre des stratégies adoptées par l'agent.

Notre prochaine étape vise à modéliser la prise de décision quant à la stratégie à adopter par l'agent dans le cas d'une non-congruence. De plus, un modèle d'actions pour mettre en place la stratégie (c) décrite ci-dessus doit être mis en place pour déterminer comment l'ACA peut changer la relation exprimée par l'utilisateur (et donc la relation perçue par l'agent).

Ce modèle fait partie intégrante du module Etat mental de l'architecture de l'ACA (Figure 1). Il permet de fournir en sortie l'attitude sociale et les intentions communicatives que l'ACA devrait exprimer étant donnée la situation d'interaction.

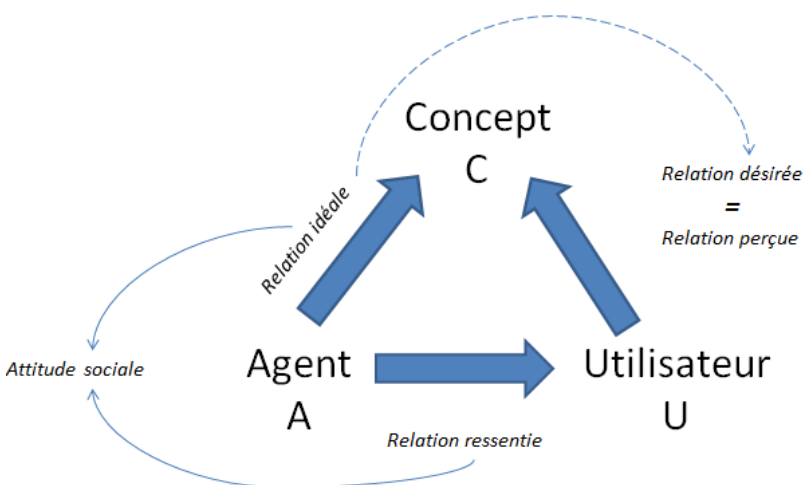

Figure 3: Exemple d'interaction congruente

\section{PERSPECTIVES DE RECHERCHE}

Dans cet article, nous avons présenté une architecture incluant les différents modèles permettant de donner la capacité à un ACA d'exprimer des émotions et des attitudes sociales à travers son comportement. La prochaine étape de nos travaux se concentrera sur l'évaluation d'un tel ACA socio-affectif. Tout d'abord, chaque modèle devra être évalué séparément pour vérifier que le comportement exprimé par l'ACA traduit en effet les émotions et attitudes sociales visées. Comme présenté dans les sections précédentes, ce type d'évaluation peut être réalisé à travers des tests perceptifs auprès d'utilisateurs [21, 24, 34]. Enfin, l'architecture dans son ensemble, i.e. avec l'ensemble des modules présentés, doit être évaluée en interaction, i.e. lorsque l'utilisateur est impliqué dans une interaction directe avec l'ACA [33]. Il s'agira alors de vérifier non seulement la perception par l'utilisateur des comportements socio-affectifs de l'ACA [34, 39] mais aussi l'effet de ces comportements sur l'engagement de l'utilisateur, sa satisfaction et ses performances dans la réalisation d'une tâche.

Les travaux présentés ci-dessus se concentrent principalement sur la modélisation du comportement socio-affectif non-verbal de l'ACA. Outre les perspectives présentées dans les sections précédentes concernant l'amélioration de chacun des modèles du comportement non-verbal, nous souhaitons intégrer dans l'ACA des modèles permettant d'étendre cette modélisation au comportement verbal à travers, d'une part, la modélisation des intentions communicatives de l'agent et d'autre part la réalisation de ces intentions, au niveau verbal. Le premier défi sera de modéliser l'influence du contexte socio-affectif sur le choix des intentions communicatives et nous travaillons actuellement sur une extension du module de dialogue DISCO [42] aujourd'hui intégré dans le module planificateur d'intentions de l'architecture présentée Figure 1. Le deuxième défi sera de déterminer comment l'ACA va exprimer verbalement ses intentions communicatives à travers le contenu linguistique (lexique, syntaxe) et paralinguistique (e.g. disfluences, 
prosodie, pause, respiration) suivant ses émotions et ses attitudes sociales et comment ces phénomènes langagiers se synchronisent avec les comportements nonverbaux présentés dans cet article.

Enfin, nous souhaitons travailler sur la compréhension du contexte de l'interaction en entrée du module Etat mental et de son modèle computationnel des attitudes sociales présenté précédemment. L'enjeu sera ici de construire un modèle socio-affectif correspondant à l'utilisateur (à partir des informations obtenues en sortie des différents modules d'analyse des signaux vidéo et audio) qui soit pertinent pour la définition de la stratégie à adopter par l'agent.

\section{REMERCIEMENTS}

Les travaux de recherche présentés dans cet article sont financés par les projets européens SSPNet, VERVE, REVERIE et TARDIS et par les projets ANR Anipev, $\mathrm{MoCa}, \mathrm{A} 1: 1$, et IMMEMO. Les auteurs de l'article tiennent à remercier André-Marie Pez et Pierre Philippe pour leur aide précieuse pour la partie développement de l'ACA.

\section{BIBLIOGRAPHIE}

1. Allwood, J., Kopp, S., Grammer, K., Ahlsen, E., Oberzaucher, E., Koppensteiner, M., The Analysis of Embodied Communicative Feedback in Multimodal Corpora: a Prerequisite for Behavior Simulation, Language Resources and Evaluation 41(3-4), 2007, p. 255-272

2. Argyle, M.: Bodily Communication. University paperbacks. Methuen, 1988.

3. Ballin, D., Gillies, M., \& Crabtree, I. A Framework For Interpersonal Attitude And Non-Verbal Communication In Improvisational Visual Media Production. In Proc. Conference on Visual Media Production, 2004, 203-210

4. Beale, R. and Creed, C. Affective interaction: How emotional agents affect users. Int. J. Hum.-Comput. Stud. 67(9): 755-776, 2009.

5. Bevacqua, E. Hyniewska, S. and Pelachaud, C. Positive influence of smile backchannels in ECAs, in Proc. of Workshop on Interacting with ECAs, AAMAS Conference, 2010.

6. Bickmore, T.W., Picard, R.W. Establishing and maintaining long-term human-computer relationships. ACM Trans. Comput.-Hum.Interact. 12(2), 2005, 293-327.

7. Burgoon, J.K., Buller, D.B., Hale, J.L., de Turck, M.A. Relational Messages Associated with Nonverbal Behaviors. Human Communication Research 10(3), 1984, 352-378.

8. Busso, C., Deng, Z., Grimm, M., Neumann, U., Narayanan, S. Rigid head motion in expressive speech animation: Analysis and synthesis. IEEE
Trans. on Audio, Speech \& Language Processing 15(3), 2007, 1075-1086.

9. Carney, D., Hall, J., LeBeau, L. Beliefs about the nonverbal expression of social power. Journal of Nonverbal Behavior 29(2), 2005, 105-123.

10.Cassell, J. More than just another pretty face: Embodied conversational interface agents. Communications of the ACM 43, 2000, 70-78.

11.Cassell, J., Pelachaud, C., Badler, N., Steedman, M., Achorn, B., Bechet, T., Douville, B., Prevost, S., Stone, M. Animated conversation: Ruled-based generation of facial expression gesture and spoken intonation for multiple conversational agents. In: Computer Graphics, 1994, 413-420.

12. Chindamo, M. Allwood, J. and Ahlsen, E. "Some suggestions for the study of stance in communication," in Proc. SocialCom Conference, 2012, 617-622.

13. Chollet,M., Ochs, M. and Pelachaud, C.: A multimodal corpus for the study of non-verbal behavior conveying interpersonal stance, Workshop Multimodal Corpora, in Proc. of IVA, 2013

14. Clavel, C., Plessier, J., Martin, J. C., Ach, L., and Morel, B, Combining facial and postural expressions of emotions in a virtual character. In Proc. IVA Conference, 2009, 287-300.

15. Coulson, M. Attributing emotion to static body postures: recognition accuracy, confusions, and viewpoint dependence. Journal of Nonverbal Behavior 28 (2), 2004, 117-139.

16. Cowie, R., Cox, C., Martin, J.-C., Batliner, A., Heylen, D., Karpouzis, K., Issues in Data Labelling, In Petta P., Pelachaud C. \& Cowie R. (Eds.) EmotionOriented Systems: The Humaine Handbook, Springer-Verlag Berlin Heidelberg, 2011, 215- 244.

17.Dael, N., Mortillaro, M., and Scherer, K. R. The Body Action and Posture Coding System (BAP): Development and Reliability. Journal of Nonverbal Behavior 36, 2012, 97-121.

18.de Rosis, F., Pelachaud, C., Poggi, I., Carofiglio, V. and de Carolis, B. From Greta's mind to her face: modeling the dynamics of affective states in a conversational embodied agent. Int. J. Hum.-Comput. Stud. 59(1-2), 2003, 81-118.

19.Dias, J., \& Paiva, A. I Want to Be Your Friend: Establishing Relations with Emotionally Intelligent Agents. To appear in Proc. of AAMAS, 2013.

20.Dias, J., \& Paiva, A. Feeling and reasoning: A computational model for emotional characters. In Proc. of EPIA, 2005, 127-140.

21.Ding. Y., Pelachaud. C., and Artière. T.: Modeling Multimodal Behaviors From Speech Prosody. In Proc. of IVA, 2013. 
22.Ekman, P and Friesen, W. V.. Unmasking the Face. A guide to recognizing emotions from facial clues. Prentice-Hall, Inc., Englewood Cliffs, New Jersey, 1975.

23.Fanelli, G., Gall, J., Romsdorfer, H., Weise, T. and Van Gool, L. A 3-D Audio-Visual Corpus of Affective Communication, IEEE Transactions on Multimedia 12(6), 2010, 591-598

24.Fourati, N. and Pelachaud, C. Toward new expressive movement characterizations, In Proc. Motion in Games 2012.

25.Gatica-Perez, D. Automatic nonverbal analysis of social interaction in small groups: A review. Image and Vision Computing 27(12), 2009, 1775-1787.

26.Gillies, M., Crabtree, I., Ballin, D.: Customisation and context for expressive behaviour in the broadband world. 2004. BT Technology Journal 22(2), 2004, 16-17.

27.Gurtman, M. B.: Exploring personality with the interpersonal circumplex. Social and Personality Psychology Compass 3 (4), 2009, 601-619.

28.Huang, J. and Pelachaud, C.. Expressive body animation pipeline for virtual agent In Proc. IVA Conference, 2012, 355-362.

29.Kihlstorm, J. and Cantor, N. Social intelligence. In Handbook of Intelligence, Cambridge University Press, 2000, 359-379.

30.Kopp, S. Krenn, B. Marsella, S. Marshall, A. N. Pelachaud, C. Pirker, H. Thrisson, K.R. and Vilhjlmsson. H. Towards a common framework for multimodal generation: The behavior markup language, In IVA Conference, 2006, 21-23.

31. Krämer, N. C. Social effects of virtual assistants. A review of empirical results with regard to communication. In Proc. IVA Conference, 2008, 507-508

32.Louwerse, M. Dale, R. Bard, E. and Jeuniaux, P. Behavior matching in multimodal communication is synchronized, Cognitive Science 36 (8), 2012, 14041426.

33. McRorie, M.; Sneddon, I.; McKeown, G.; Bevacqua, E.; de Sevin, E.; Pelachaud, C., Evaluation of Four Designed Virtual Agent Personalities, IEEE Transactions on Affective Computing 3 (3), 311-322, July-September 2012

34.Ochs, M. Niewiadomski, R. Brunet, P. and Pelachaud C., Smiling Virtual Agent in Social Context, Cognitive Processing, Special Issue on "Social Agents. From Theory to Applications" 13 (22), 2012, 519-53.

35.Ochs, M. Prépin, P. and Pelachaud, C. From emotions to interpersonal stances: Multi-levels analysis of smiling virtual characters, in Proc. of ACII, 2013
36.Osgood, C., \& Tannenbaum, P. The Principle of Congruity in the Prediction of Attitude Change. Psychological Review 62(1), 1955, 42-55.

37.Pandzic, I., Forcheimer, R.: MPEG4 Facial Animation - The standard, implementations and applications. John Wiley \& Sons. 2002.

38.Prendinger, H., Descamps, S., \& Ishizuka, M. Scripting affective communication with life-like characters in web-based interaction systems. Applied Artificial Intelligence 16 (7-8), 2002, 519-553.

39.Prepin, K. Ochs, M. and Pelachaud, C. Beyond backchannels: co-construction of dyadic stance by reciprocal reinforcement of smiles between virtual agents, International Conference CogSci. 2013.

40.Raven, B.H. The Bases of Power and the Power/Interaction Model of Interpersonal Influence. Analyses of Social Issues and Public Policy 8(1), 2008, 1-22

41.Ravenet, B., Ochs, M. and Pelachaud, C. From a User-Created Corpus of Virtual Agent's Non-Verbal Behavior to a Computational Model of Interpersonal Attitudes, in Proc. of IVA, 2013.

42. Rich, C. and Sidner, C. Using collaborative discourse theory to partially automate dialogue tree authoring In Proc. of IVA Conference. 2012, 327-340.

43. Roether, C. L., Omlor, L., Christensen, A., and Giese, M. A. Critical features for the perception of emotion from gait. Journal of Vision 9 (6), 2009, 1-32.

44.Sadler, P., Ethier, N., \& Woody, E. (2011). Interpersonal Complementarity. Dans Handbook of interpersonal psychology: Theory, research, assessment, and therapeutic interventions, New York: Horowitz, L.M. ; Strack, S, 2011, 123-142.

45.Salovey P., Bedell B., Detweiler J., Mayer J., « Current Directions in Emotional Intelligence Research », Lewis M., Haviland-Jones J., Eds., Handbook of Emotions, The Guilford Press, New York, 2000, 504-520

46. Scherer, K. "What are emotions? And how can they be measured?" Social Science Information 4(4), 2005, 685-729.

47.Trapnell, P., \& Wiggins, J. (1990). Extension of the Interpersonal Adjective Scales to Include the Big Five Dimensions of Personality. Journal of Personality and Social Psychology 59 (4), 1990 , p.781-790.

48.Wallbott, H. G. (1998). Bodily expression of emotion. European Journal of Social Psychology, 28(6), 879-896. 